\title{
Trap-Free Hot Carrier Relaxation in Lead-Halide Perovskite Films
}

Simon A. Bretschneider ${ }^{1}$, Frédéric Laquai ${ }^{2}$ and Mischa Bonn ${ }^{1 *}$

${ }^{1}$ Department of Molecular Spectroscopy, Max Planck Institute for Polymer Research, Ackermannweg 10, 55128 Mainz, Germany

${ }^{2}$ King Abdullah University of Science and Technology (KAUST), KAUST Solar Center (KSC), Physical Sciences and Engineering Division (PSE), Material Science and Engineering Program (MSE), Thuwal, 23955-6900, Kingdom of Saudi Arabia

\section{Corresponding Author}

Mischa Bonn, bonn@mpip-mainz.mpg.de,+49 6131379161 


\begin{abstract}
Photovoltaic devices that employ lead-halide perovskites as photoactive materials exhibit power conversion efficiencies of $22 \%$. One of the potential routes to go beyond the current efficiencies is to extract charge carriers that carry excess energy, that is, non-relaxed or 'hot' carriers, before relaxation to the band minima is completed. Lead-halide perovskites have been demonstrated to exhibit hot-carrier relaxation times exceeding 100 picoseconds for both single- and polycrystalline samples. Here, we demonstrate, using a combined time-resolved photoluminescence and transient absorption study supported by basic modelling of the dynamics, that the decay of the high-energy part of the photoluminescence occurs on a timescale $(\sim 100 \mathrm{ps})$ very similar to the repopulation of the band minima when excited with a photon energy larger than $2.6 \mathrm{eV}$. The similarity between the two timescales indicates that the depopulation of hot states occurs without transient trapping of electrons or holes.
\end{abstract}




\section{INTRODUCTION}

Since organic-inorganic lead-halide perovskites were proposed for solar cell applications by Kojima et al. in 2009, the scientific community has witnessed great progress of both, device performance and fundamental understanding of the photophysical processes following their photoexcitation. ${ }^{1-7}$ However, although perovskite-based solar cells have now exceeded power conversion efficiencies of $21.5 \%$, some of their fundamental physical properties such as the nature of the band-gap and occurrence of ferroelectricity, are still under debate. ${ }^{8-14}$ Intense research has also been dedicated to study non-relaxed, hot, carriers in $\mathrm{CH}_{3} \mathrm{NH}_{3} \mathrm{PbI}_{3}\left(\mathrm{MAPbI}_{3}\right)$. In principle, reducing the losses associated with thermalization of hot carriers could allow the solar cell efficiency to surpass the Shockley-Queisser limit. In fact, if hot carriers could be extracted prior to their relaxation and recombination, then the maximum obtainable power conversion efficiency could theoretically be as high as $44 \% .^{15,16}$ For $\mathrm{MAPbI}_{3}$, long-lived ( $\geq 50 \mathrm{ps}$ ) hot carriers have been reported in two pump fluence-dependent regimes: at high $\left(>10^{18} / \mathrm{cm}^{3}\right)$ charge carrier densities a "hot-phonon bottleneck" was observed that slows down cooling of hot carriers. The hot-phonon bottleneck originates from charge carrier reheating due to reabsorption of phonons. ${ }^{17,18}$ At lower $\left(\leq 10^{17} / \mathrm{cm}^{3}\right)$ charge carrier densities, high-energy photoluminescence originates from carriers that carry a large excess energy of up to $0.25 \mathrm{eV}$. This effect has been attributed to carrier screening resulting from the reorientation of the polar cations in the perovskite lattice. ${ }^{16,19}$ Niesner et al. have reported a rise of the integrated signal intensity in timeresolved two-photon photoelectron (2-PPE) spectroscopy for more than $60 \mathrm{ps}$ attributed to photoelectrons close to the conduction band minimum, in conjunction with a simultaneous decay of the intensity of high-energy photoelectrons. Manser and Flender et al. have observed a twocomponent decay of a population of hot carriers at $\sim 2.5 \mathrm{eV}$ using transient absorption 
spectroscopy. The fast, sub-ps component of this decay was attributed to relaxation of hot holes towards the band-minima. ${ }^{20}$ The slow component with a lifetime of $\sim 50$ ps was interpreted as slow thermalization of carriers from the $\mathrm{M}$ - to the $\Gamma$-point in the energy band structure of $\mathrm{MAPbI}_{3}{ }^{20-27}$ Here, we investigate hot carrier dynamics in solution-processed polycrystalline $\mathrm{MAPbI}_{3}(\mathrm{Cl})$ perovskite films with lower excess energy $(\leq 0.15 \mathrm{eV})$ by time-resolved photoluminescence (PL) and transient absorption (TA) spectroscopy. We find high-energy photoluminescence in solution-processed $\mathrm{MAPbI}_{3}(\mathrm{Cl})$ exhibiting a relaxation rate of $0.16 \mathrm{meV} / \mathrm{ps}$ for excess energies exceeding $0.15 \mathrm{eV}$ when excited at $3.1 \mathrm{eV}$. We infer lifetime of $94 \pm 13 \mathrm{ps}$ for the spectrally-integrated fraction of the photoluminescence above energies of 1.7. From TA experiments, the signal of the ground-state bleach (GSB) is observed to peak at $100 \pm 5$ ps when excited above $2.6 \mathrm{eV}$. The similarity of the timescales clearly indicates that the hot carrier thermalization occurs without transient trapping of charge carriers.

\section{METHODS}

The perovskite samples were prepared in a nitrogen-filled glovebox with an oxygen level of $<$ 1ppm. A $40 \mathrm{wt} \%$ solution of MAI (prepared following Lee et al., see Ref. 2) and $\mathrm{PbCl}_{2}(99.999 \%$ purity, Sigma Aldrich; dehydrated under high vacuum conditions) in anhydrous DMF (Sigma Aldrich) was spin-coated on fused silica for $60 \mathrm{~s}$ at $2000 \mathrm{rpm}$ with a molar ration of 3:1 $\mathrm{MAI}: \mathrm{PbCl}_{2}$. After a room temperature annealing for 30 minutes, the samples were annealed at $100^{\circ} \mathrm{C}$ for 60 minutes. The samples thickness $(422 \pm 26 \mathrm{~nm})$ was measured using a KLA Tencor P16+ step profiler at six different positions of the sample. The samples were stored overnight prior to analysis. Transient absorption and photoluminescence measurements were performed under high vacuum conditions without any exposure to ambient conditions at $291 \mathrm{~K}$. 
Time-resolved photoluminescence was measured with a C5680 Hamamatsu streak camera system following excitation at $400 \mathrm{~nm}$ from a frequency-doubled mode-locked titanium:sapphire amplifier (Coherent LIBRA HE, $3.5 \mathrm{~mJ}, 100 \mathrm{fs}$ ), either with a repetition rate of $1 \mathrm{kHz}$ (10 ns time window; instrument response function $81 \mathrm{ps}$ ) or with a repetition rate of $80 \mathrm{MHz}$ (2 ns time window; instrument response function $16 \mathrm{ps}$ ).

Transient absorption experiments were carried out with a home-built pump-probe setup. The output of a titanium:sapphire amplifier was used to seed two independent optical parametric amplifiers (Coherent OPerA Solo), of which one was used to generate a pump pulse. The second OPA was used generate a seed pulse for supercontinuum generation, which served as the broadband probe pulse. For supercontinuum generation in the range of 550-1000 nm, a $1300 \mathrm{~nm}$ pulse was focused into a $3 \mathrm{~mm}$ c-cut sapphire crystal. Time resolution was obtained by delaying the pump pulses on a motorized delay stage between $300 \mathrm{fs}$ and $8 \mathrm{~ns}$ with respect to the probe pulse. The transmission spectrum of the probe pulses was measured with a linear silicon photodiode array and successive probe pulses were used to determine the change in transmission induced by the pump pulse. Data collection and analysis were performed with home-built readout electronics and a LabView-based data acquisition and analysis software.

UV/VIS measurements were performed using a Perkin Elmer UV/VIS/NIR spectrometer (Lambda 900) in a $150 \mathrm{~mm}$ integrating sphere with a spectral resolution of $1 \mathrm{~nm}$.

Scanning electron microscopy images were measured using a Zeiss LEO Gemini 1530. 


\section{RESULTS \& DISCUSSION}

To investigate the dynamics of hot carriers in $\mathrm{MAPbI}_{3}(\mathrm{Cl})$, we studied both the radiative recombination (via PL spectroscopy) and population dynamics of excited states (using TA spectroscopy). The samples were prepared by spin-coating $\mathrm{MAI}$ and $\mathrm{PbCl}_{2}$ from solution in $\mathrm{DMF}$ on fused silica and subsequent thermal annealing in a nitrogen-filled glovebox. The preparation results in polycrystalline films with micrometer-sized crystals (compare Fig. S2) with a thickness of $\sim 420 \mathrm{~nm}$. Both, transient absorption and photoluminescence measurements were performed under high dynamic vacuum conditions $\left(\mathrm{p}<5 \cdot 10^{-6} \mathrm{mbar}\right)$ at $291 \mathrm{~K}$.

The occurrence of long-lived hot carriers was investigated using TA measurements with excitation at $3.1,2.6$ and $2.05 \mathrm{eV}$ with low $\left(2.5 \pm 0.25 \mu \mathrm{J} / \mathrm{cm}^{2}\right)$ fluence. Fig. 1, (A) shows a false color two-dimensional map of TA measurement after excitation at $3.1 \mathrm{eV}$ obtained at a fluence of $2.5 \mu \mathrm{J} / \mathrm{cm}^{2}$. 
(A)

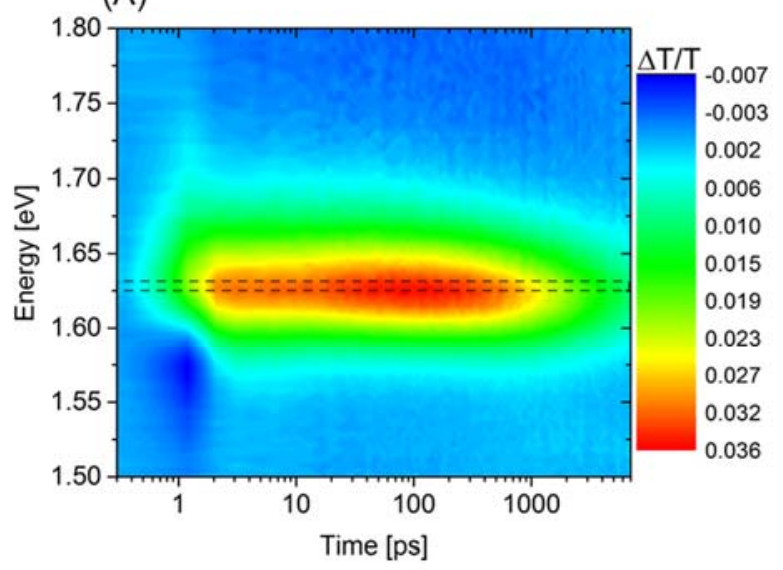

(C)

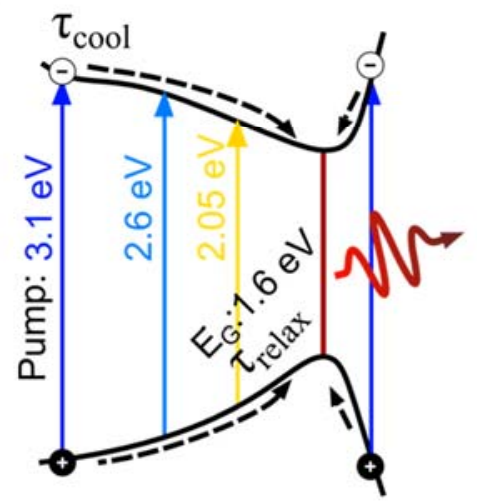

(B)

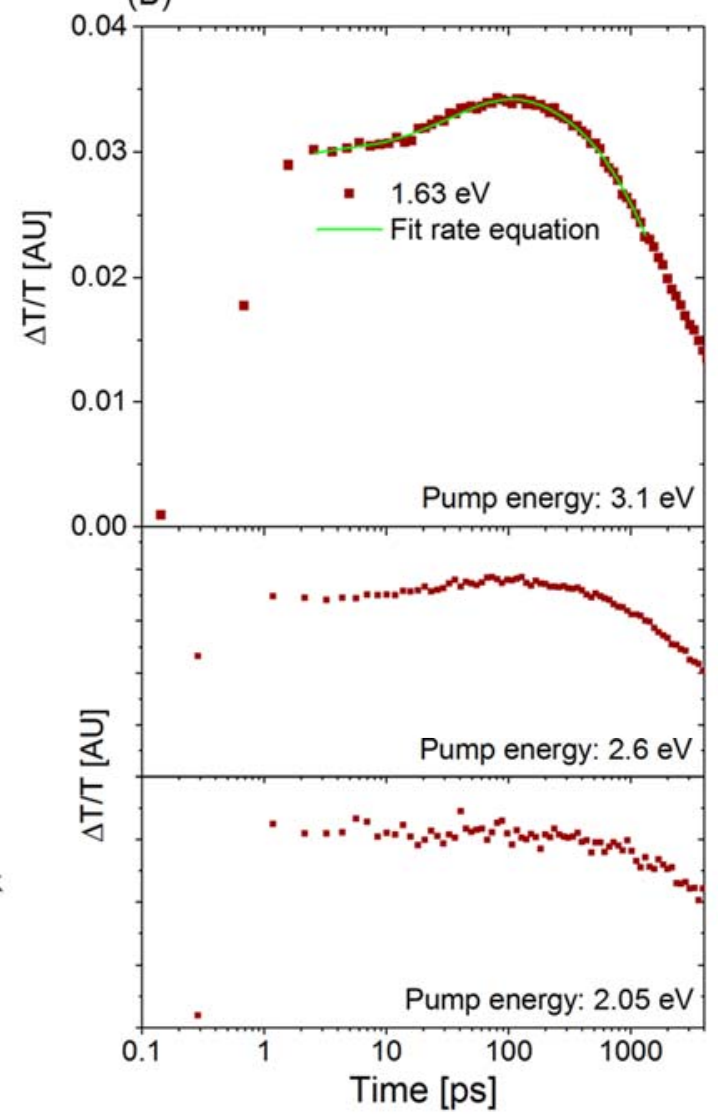

Figure 1: ps-ns transient absorption measurements on $\mathrm{MAPbI}_{3}(\mathrm{Cl})$ films after excitation with a $\sim 150$ fs pulse with a fluence of $2.5 \pm 0.25 \mu \mathrm{J} / \mathrm{cm}^{2}$. (A) shows a contour plot of the transient absorption data after excitation at $3.1 \mathrm{eV}$. (B) shows the kinetics of the GSB probed at $1.63 \pm$ $0.01 \mathrm{eV}$ (brown, as indicated by the dashed box in (A)) for different pump photon energies. For a pump photon energy of $3.1 \mathrm{eV}$ the maximum of the bleach increases by $11 \%$ on a timescale of $100 \pm 5$ ps (upper panel). The fit obtained from the rate equation is shown in green (fitting parameters in the SI). For a pump photon energy of $2.6 \mathrm{eV}$ (middle panel), the bleach increases similar to the case of excitation at $3.1 \mathrm{eV}$. For a pump photon energy of $2.05 \mathrm{eV}$ (lower panel), no further growth of the bleach can be observed, the bleach remains flat for $\sim 200$ ps. (C) shows a scheme of the energy levels relevant to understand the TA measurements and the simplified three-level model used for the rate equations. The populations of the three energy levels (ground 
state, band edges and hot states) are denoted as $\mathrm{N}_{\mathrm{GS}}, \mathrm{N}_{\mathrm{BE}}$ and $\mathrm{N}_{\text {hot }}$; their respective lifetimes are $\tau_{\text {relax }}$ and $\tau_{\text {cool }}$

The broad feature centered at $1.63 \mathrm{eV}$ is commonly assigned to the ground-state bleach (GSB) related to the transition between the valence band (VB) maximum and the conduction band (CB) minimum at the $\Gamma$-point in the energy band diagram. ${ }^{3,}{ }^{28}$ The derivative-like feature between 1.5 and $1.6 \mathrm{eV}$ at $1 \mathrm{ps}$, partially overlapping with the GSB, was previously assigned to photoinduced reflectivity changes. ${ }^{18}$ The high-energy tail above $1.7 \mathrm{eV}$, which exhibits a sub-ps lifetime, can be associated with a population of hot carriers. ${ }^{17,18,20}$ Kinetics extracted from the two-dimensional plot are shown in Fig. 1, (B). The integrated signal (probed at $1.63 \pm 0.01 \mathrm{eV}$ ) rises within the instrument response time of $\sim 1$ ps to an amplitude of 0.03 . Furthermore, the signal subsequently increases to a value of 0.034 within $100 \pm 5$ ps. The dynamics of the GSB after excitation at 2.6 $\mathrm{eV}$ show a similar trend. These results indicate that excitation at $3.1 \mathrm{eV}$ and $2.6 \mathrm{eV}$ leads to electrons and holes populating energetically high-lying states far from the VB maximum and CB minimum, as illustrated in Fig. 1(C). Relaxation from these high-lying states to the $\Gamma$-point leads to a rise in the TA signal at $1.63 \mathrm{eV}$. For excitation at $2.05 \mathrm{eV}$, the GSB signal does not show a subsequent increase but rather stays constant before the population decays.

In order to quantify the energy-dependent kinetics of the GSB we approximate the kinetics using a simple three-level system, also shown in Fig. 1(C). The pump excites charge carriers from the ground to the excited states; an electron-hole pair is generated. We implemented the sub-ps carrier thermalization resulting in direct population of the band-edge state, $\mathrm{N}_{\mathrm{BE}}$. A fraction of the carriers does not undergo immediate thermalization, but forms a hot population, $\mathrm{N}_{\text {hot, }}$ relaxing to the band-edge state with a cooling time, $\tau_{\text {cool. }}$ The band edge population, NBE decays back to the 


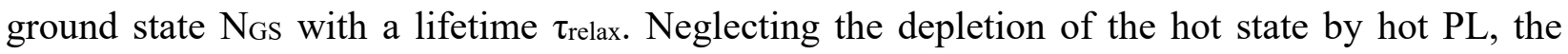
resulting rate equations read:

$$
\begin{array}{rlrl}
\frac{\mathrm{dN}}{\mathrm{dt} S} & =\frac{1}{\tau_{\text {relax }}} \mathrm{N}_{\mathrm{BE}}(\mathrm{t}) & \frac{\mathrm{dN} \mathrm{BE}_{\mathrm{B}}}{\mathrm{dt}}=\frac{1}{\tau_{\text {cool }}} \mathrm{N}_{\text {hot }}(\mathrm{t})-\frac{1}{\tau_{\text {relax }}} \mathrm{N}_{\mathrm{BE}}(\mathrm{t}) \\
\frac{\mathrm{dN} \text { hot }}{\mathrm{dt}}=-\frac{1}{\tau_{\text {cool }}} \mathrm{N}_{\text {hot }}(\mathrm{t}) & \mathrm{N}_{\mathrm{GS}}+\mathrm{N}_{\mathrm{BE}}+\mathrm{N}_{\text {hot }}=1
\end{array}
$$

The GSB TA signal $\Delta \mathrm{T} / \mathrm{T}$ is proportional to $\left(1-\left(\mathrm{N}_{\mathrm{GS}}-\mathrm{N}_{\mathrm{BE}}\right)\right)$. Immediately after excitation, the initial conditions are $\mathrm{N}_{\text {hot }}(0)=\delta$ and $\mathrm{N}_{\mathrm{BE}}(0)=\gamma$, with $\delta, \gamma \ll 1$. The values for $\delta, \gamma, \tau_{\text {relax }}$ and $\tau_{\text {cool }}$ were adjusted to provide best description of the data between 2.5 and 1000 ps. The result is shown in Fig. 1, (B); the kinetics of the population of the band edges and hot population are shown in Fig. S3. The fit follows the bleach signal probed at $1.63 \mathrm{eV}$, peaking at 108 ps. Fitting to the rate equations resulted in a cooling time of the hot population of $40 \pm 2$ ps. For a fluence of $12.5 \mu \mathrm{J} / \mathrm{cm}^{2}$, the lifetime of the bleach bands is reduced as expected for fast nongeminate recombination due to the high initial charge carrier density (see Fig. S4). For a fluence of $39 \mu \mathrm{J} / \mathrm{cm}^{2}$, we observed signatures of a hot-phonon bottleneck (see Fig. S5) in agreement with previous results published measurements by Yang and Price et al., confirming the equivalence of our results to previously published data. ${ }^{17,18}$

In addition to the investigation of the population of excited states, which is accessible by TA spectroscopy, the radiative recombination of charge carriers was measured by time-resolved photoluminescence spectroscopy using the Streak Camera technique. Figure 2, (A) shows a false color two-dimensional plot of the photoluminescence measured over a time-window of $10 \mathrm{~ns}$ on a logarithmic intensity scale. 

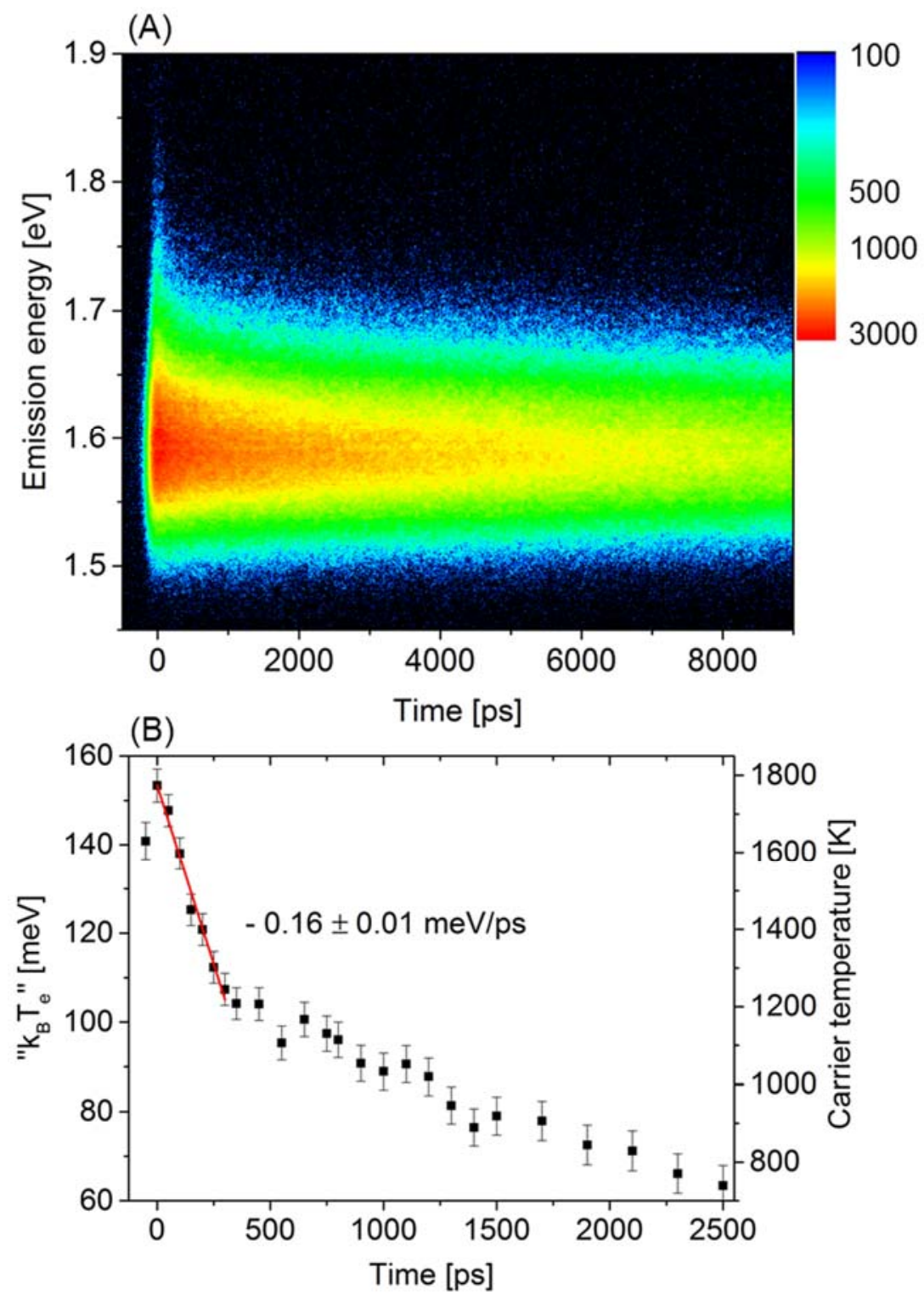

Figure 2: Time-resolved photoluminescence measurements on $\mathrm{MAPbI}_{3}(\mathrm{Cl})$ films after excitation with a 100 fs pulse at $3.1 \mathrm{eV}$ with a fluence of $2.5 \mu \mathrm{J} / \mathrm{cm}^{2}$. (A) shows the photoluminescence contour plot. The high energy tail is associated with the recombination of non-relaxed, 'hot', charge carriers visible at early timescales. (B) shows the temporal evolution (carrier cooling) of the excess energy/effective carrier temperatures $\mathrm{k}_{\mathrm{B}} \mathrm{T}_{\mathrm{e}}$ obtained from a global fit of the data according to a model proposed by $\mathrm{Zhu}^{19}$. The lifetime of the initial fast decay was approximated by a linear fit (red solid line) with a slope of $-0.16 \pm 0.01 \mathrm{meV} / \mathrm{ps}$. 
The PL response is dominated by the band-edge emission centered at $1.59 \mathrm{eV}$. In addition to the band-edge emission, a high-energy tail is present between 1.7 and $1.85 \mathrm{eV}$. This tail of the PL does not originate from high signal intensities at short time scales, as evident from different normalized spectra (see Fig. S6, (B)). The high-energy tail appears to originate from photoluminescence of hot charge carriers, in agreement with previous reports by Zhu and Niesner et al. ${ }^{16,19}$ To analyze the excess energy of the states from which the high-energy photon emission occurs, we performed a spectral deconvolution based on a global fit of the spectra between -50 and 2500 ps relative to the maximum emission signal intensity. This procedure allows extraction of the excess energy with an effective carrier temperature, $k_{B} T_{e}$, based on the model proposed by Zhu et al.. ${ }^{19}$ After the initial charge carrier generation, the effective carrier temperature (compare Fig. 2B) rises to $1780 \pm 43 \mathrm{~K}$, equivalent to an excess energy of $153 \pm 4 \mathrm{meV}$. The initial decay of $\sim 60 \mathrm{meV}$ within $300 \mathrm{ps}$ can be attributed to the decay of the high-energy photoluminescence. The relaxation rate for this decay is $0.16 \pm 0.01 \mathrm{meV} / \mathrm{ps}$, which is comparable to the values previously observed by Niesner et al.. ${ }^{16}$ For an estimate of the lifetime of the high energy photoluminescence, we compared the spectrally integrated total photoluminescence signal amplitude to the fraction of the photoluminescence signal above $1.7 \mathrm{eV}$ (compare Fig. S6, C). We fit the signal decay with a single exponential function and obtained lifetime of $94 \pm 13$ ps. For the photoluminescence signal probed at $1.7 \mathrm{eV}$ (see Fig. S6, D), we obtained a lifetime of $117 \pm 10$ ps. For comparison, the fast decay of the initial "hot" effective carrier temperatures $\mathrm{k}_{\mathrm{B}} \mathrm{T}_{\mathrm{e}}$ has a half-life of $\sim 140$ ps. It should be noted that the amount of excess energy also depends on the position of the quasi Fermi levels. This increases the uncertainty of the value we determine, but not necessarily for the dynamics of the decay of the excess energy. ${ }^{16,22,29}$

The very similar timescales of the decay of the high-energy photoluminescence $(\sim 100-140 \mathrm{ps})$ and the bleach signal reaching completion $(\sim 100 \mathrm{ps}$, with a $\sim 40 \mathrm{ps}$ rise time $)$ point to both 
effects sharing the same physical origin. The appearance of the ground state population virtually in parallel with the decay of the high-energy photoluminescence, points to direct relaxation from high-energy states to the $\Gamma$-point. If hot charge carriers underwent transient trapping while relaxing to the band edges, the band edge population would not rise as quickly as observed, but instead the buildup would take even longer. Furthermore, it is also questionable whether, in such a case, the simple three-level model would still provide an adequate description of the dynamics, as it does in our experiments. ${ }^{30}$ We attribute the slight red-shift of the GSB after 100 ps to a (although weak) signature of a dynamic Burstein-Moss-shift. ${ }^{17,18,23}$ The photon energy-dependent TA measurements allow assumptions about the origin of the hot carriers. The delayed population of the ground state can be observed for excitation above $2.6 \mathrm{eV}$, but not for excitation at $2.05 \mathrm{eV}$, where it replicates published dynamics of perovskites films prepared from lead-chloride. ${ }^{31,32}$ Due to the limited probe range in our TA measurement we cannot probe the origin of the hot carriers directly. A tentative proposition for the origin of the hot carriers based on the photon energydependent TA measurements suggests a long-lived population at the M-point of the Brillouin zone. $^{3,21,23-25}$ Niesner et al. have shown a decay of the intensity of photoelectrons with an excess energy of $\sim 0.25 \mathrm{eV}$ and a subsequent increase of the photoelectron intensity associated with a population close to the Fermi energy on a similar timescale as in our TA measurements. As such, our results validate those conclusions, but there are some notable differences between the experiments: TA measurements described above probe the bulk sample in transmission, and were carried out at room temperature, rather than being surface sensitive as 2-PPE spectroscopy and being measured at $190 \mathrm{~K}$. 2-PPE also is sensitive to photoelectrons only, whereas TA probes both electrons and holes. Furthermore, a recently study by Guo et al. using spatially-resolved and pump-photon energy dependent transient absorption spectroscopy on $\mathrm{MAPbI}_{3}$ films showed a similar slow rise of the GSB signal for a pump photon energy of $3.14 \mathrm{eV}$, while it was absent for 
a pump photon energy of $1.68 \mathrm{eV}$ in excellent agreement with our data presented in Figure $1 .{ }^{27}$ In fact, Guo et al. provide clear evidence for quasi-ballistic transport of hot charge carriers, which supports our conclusion that trapping effects are negligible for hot carriers excited with pump photon energies above $2.6 \mathrm{eV}$.

The high-energy photoluminescence also originates from the hot carrier population. The exact mechanism of the high-energy photoluminescence itself is still under debate. Wehrenfennig et al. have proposed dynamic spectral broadening due to carrier-phonon interaction. ${ }^{33}$ Chang et al. have proposed a transient change of the recombination rates with a characteristic timescale of $<$ 80 ps resulting from the emission of high-energy phonons (of the MA cations) and subsequent phonon-phonon coupling. ${ }^{34}$ A transient change of the recombination rate caused by a long-lived population of hot carriers in combination with excitation of MA phonon modes could also explain the absence of a bleach signal in the TA measurements associated with the high-energy photoluminescence. In fact, it would also explain the lack of high-energy photoluminescence from $\mathrm{CsPbI}_{3}$ as reported by $\mathrm{Zhu}$ et al.. ${ }^{19}$ Different mechanisms could contribute to the rise of the GSB, including interband relaxation from a higher-lying conduction band to the lowest-energy conduction band and/or intervalley scattering. ${ }^{3,16,20,25}$ To better understand the origin of the various hot carrier processes, additional experiments are clearly required; specifically, the question whether polarons, as proposed by Zhu et $a{ }^{35}$ are responsible for the low carrier scattering rates or whether a proposed spin-split indirect band gap can explain the remarkable hot carrier properties. ${ }^{9,10,36}$ 


\section{CONCLUSIONS}

In conclusion, we report high-energy photoluminescence and long-lived hot charge carriers in polycrystalline solution-processed $\mathrm{MAPbI}_{3}(\mathrm{Cl})$ films similar to previous reports on co-evaporated $\mathrm{MAPbI}_{3}$ and $\mathrm{MAPbBr}_{3}$ single crystals. ${ }^{16,19}$ Our results suggest that the high-energy photoluminescence, which exhibits an excess energy of up to $0.15 \mathrm{eV}$, is correlated with a delayed population of the band edges with charge carriers after excitation with a photon energy larger than $2.6 \mathrm{eV}$. The similar timescales of the hot carrier relaxation and the increased population of the band edges indicate a direct depopulation of hot states without transient trapping of charge carriers during the cooling process.

\section{SUPPORTING INFORMATION}

Optical characterization and $\mathrm{SEM}$ of $\mathrm{MAPbI}_{3}(\mathrm{Cl})$, additional transient absorption and photoluminescence measurements.

\section{Notes}

The authors declare no competing financial interest.

\section{Author Contributions}

The manuscript was written through contributions of all authors. All authors have given approval to the final version of the manuscript. 


\section{ACKNOWLEDGEMENTS}

We thank Enrique Canovas, Felix Deschler, Mike Donovan, Dominik Gehrig, Ian Howard, Sapun Parekh, Hai Wang and Stefan Weber for fruitful discussions and Jenée Cyran for a careful reading of the manuscript. S.A.B acknowledges support from the Max Planck Graduate Center, thanks Stefan Schumacher for the TOC image, Gunnar Glaser for providing wisdom, especially about scanning electron microscopy and Ellen Backus for help with the simulations. F. L. thanks the Max-Planck-Society for previous funding of the Max Planck Research Group for Organic Optoelectronics.

\section{REFERENCES}

1. Kojima, A.; Teshima, K.; Shirai, Y.; Miyasaka, T. Organometal Halide Perovskites as Visible-Light Sensitizers for Photovoltaic Cells. J. Am. Chem. Soc. 2009, 131, 6050-6051. 2. Lee, M. M.; Teuscher, J.; Miyasaka, T.; Murakami, T. N.; Snaith, H. J. Efficient Hybrid Solar Cells Based on Meso-Superstructured Organometal Halide Perovskites. Science 2012, 338, 643-647.

3. Herz, L. M. Charge-Carrier Dynamics in Organic-Inorganic Metal Halide Perovskites. Annu. Rev. Phys. Chem. 2016, 67, 65-89.

4. Stranks, S. D.; Snaith, H. J. Metal-Halide Perovskites for Photovoltaic and Light-Emitting Devices. Nat. Nanotechnol. 2015, 10, 391-402.

5. Zhang, W.; Eperon, G. E.; Snaith, H. J. Metal Halide Perovskites for Energy Applications. Nat. Energy 2016, 1, 16048.

6. $\quad$ Li, W.; Wang, Z.; Deschler, F.; Gao, S.; Friend, R. H.; Cheetham, A. K. Chemically Diverse and Multifunctional Hybrid Organic-Inorganic Perovskites. Nat. Rev. Mater. 2017, 2 , 16099.

7. Bretschneider, S. A.; Weickert, J.; Dorman, J. A.; Schmidt-Mende, L. Research Update: Physical and Electrical Characteristics of Lead Halide Perovskites for Solar Cell Applications. APL Mater. 2014, 2, 040701.

8. $\quad$ Saliba, M.; Matsui, T.; Domanski, K.; Seo, J.-Y.; Ummadisingu, A.; Zakeeruddin, S. M.; Correa-Baena, J.-P.; Tress, W. R.; Abate, A.; Hagfeldt, A. et al. Incorporation of Rubidium Cations into Perovskite Solar Cells Improves Photovoltaic Performance. Science 2016, 354, 206209.

9. Hutter, E. M.; Gelvez-Rueda, M. C.; Osherov, A.; Bulovic, V.; Grozema, F. C.; Stranks, S. D.; Savenije, T. J. Direct-Indirect Character of the Bandgap in Methylammonium Lead Iodide Perovskite. Nat. Mater. 2017, 16, 115-120. 
10. Azarhoosh, P.; McKechnie, S.; Frost, J. M.; Walsh, A.; van Schilfgaarde, M. Research Update: Relativistic Origin of Slow Electron-Hole Recombination in Hybrid Halide Perovskite Solar Cells. APL Mater. 2016, 4, 091501.

11. Cho, K. T.; Paek, S.; Grancini, G.; Roldan Carmona, C.; Gao, P.; Lee, Y. H.; Nazeeruddin, M. K. Highly Efficient Perovskite Solar Cells with a Compositional Engineered Perovskite/Hole Transporting Material Interface. Energy \& Environmental Science 2017, 10, 621-627.

12. Hermes, I. M.; Bretschneider, S. A.; Bergmann, V. W.; Li, D.; Klasen, A.; Mars, J.; Tremel, W.; Laquai, F.; Butt, H.-J.; Mezger, M. et al. Ferroelastic Fingerprints in Methylammonium Lead Iodide Perovskite. J. Phys. Chem. C 2016, 120, 5724-5731.

13. Kutes, Y.; Ye, L.; Zhou, Y.; Pang, S.; Huey, B. D.; Padture, N. P. Direct Observation of Ferroelectric Domains in Solution-Processed $\mathrm{CH}_{3} \mathrm{NH}_{3} \mathrm{PbI}_{3}$ Perovskite Thin Films. J. Phys. Chem. Lett. 2014, 5, 3335-3339.

14. Tress, W. Perovskite Solar Cells on the Way to Their Radiative Efficiency Limit Insights into a Success Story of High Open-Circuit Voltage and Low Recombination. Adv. Energy Mater. 2017, 1602358.

15. Green, M., Third Generation Photovoltaics; Springer-Verlag Berlin Heidelberg, 2003.

16. Niesner, D.; Zhu, H.; Miyata, K.; Joshi, P. P.; Evans, T. J. S.; Kudisch, B. J.; Trinh, M. T.; Marks, M.; Zhu, X. Persistent Energetic Electrons in Methylammonium Lead Iodide Perovskite Thin Films. J. Am. Chem. Soc. 2016, 138, 15717-15726.

17. Yang, Y.; Ostrowski, D. P.; France, R. M.; Zhu, K.; van de Lagemaat, J.; Luther, J. M.; Beard, M. C. Observation of a Hot-Phonon Bottleneck in Lead-Iodide Perovskites. Nat. Photonics 2015, 10, 53-59.

18. Price, M. B.; Butkus, J.; Jellicoe, T. C.; Sadhanala, A.; Briane, A.; Halpert, J. E.; Broch, K.; Hodgkiss, J. M.; Friend, R. H.; Deschler, F. Hot-Carrier Cooling and Photoinduced Refractive Index Changes in Organic-Inorganic Lead Halide Perovskites. Nat. Commun. 2015, 6, 8420.

19. Zhu, H.; Miyata, K.; Fu, Y.; Wang, J.; Joshi, P. P.; Niesner, D.; Williams, K. W.; Jin, S.; Zhu, X.-Y. Screening in Crystalline Liquids Protects Energetic Carriers in Hybrid Perovskites. Science 2016, 353, 1409-1413.

20. Xing, G.; Mathews, N.; Sun, S.; Lim, S. S.; Lam, Y. M.; Grätzel, M.; Mhaisalkar, S.; Sum, T. C. Long-Range Balanced Electron- and Hole-Transport Lengths in Organic-Inorganic $\mathrm{CH}_{3} \mathrm{NH}_{3} \mathrm{PbI}_{3}$. Science 2013, 342, 344-347.

21. Flender, O.; Klein, J. R.; Lenzer, T.; Oum, K. Ultrafast Photoinduced Dynamics of the Organolead Trihalide Perovskite $\mathrm{CH}_{3} \mathrm{NH}_{3} \mathrm{PbI}_{3}$ on Mesoporous $\mathrm{TiO}_{2}$ Scaffolds in the 320-920 nm Range. Phys. Chem. Chem. Phys. 2015, 17, 19238-19246.

22. Kawai, H.; Giorgi, G.; Marini, A.; Yamashita, K. The Mechanism of Slow Hot-Hole Cooling in Lead-Iodide Perovskite: First-Principles Calculation on Carrier Lifetime from Electron-Phonon Interaction. Nano Lett. 2015, 15, 3103-3108.

23. Manser, J. S.; Kamat, P. V. Band Filling with Free Charge Carriers in Organometal Halide Perovskites. Nat. Photonics 2014, 8, 737-743.

24. Leguy, A. M. A.; Azarhoosh, P.; Alonso, M. I.; Campoy-Quiles, M.; Weber, O. J.; Yao, J.; Bryant, D.; Weller, M. T.; Nelson, J.; Walsh, A. et al. Experimental and Theoretical Optical Properties of Methylammonium Lead Halide Perovskites. Nanoscale 2016, 8, 6317-6327. 25. Anand, B.; Sampat, S.; Danilov, E. O.; Peng, W.; Rupich, S. M.; Chabal, Y. J.; Gartstein, Y. N.; Malko, A. V. Broadband Transient Absorption Study of Photoexcitations in Lead Halide Perovskites: Towards a Multiband Picture. Phys. Rev. B 2016, 93, 161205. 
26. Amat, A.; Mosconi, E.; Ronca, E.; Quarti, C.; Umari, P.; Nazeeruddin, M. K.; Grätzel, M.; De Angelis, F. Cation-Induced Band-Gap Tuning in Organohalide Perovskites: Interplay of Spin-Orbit Coupling and Octahedra Tilting. Nano Lett. 2014, 14, 3608-3616.

27. Guo, Z.; Wan, Y.; Yang, M.; Snaider, J.; Zhu, K.; Huang, L. Long-Range Hot-Carrier Transport in Hybrid Perovskites Visualized by Ultrafast Microscopy. Science 2017, 356, 59-62. 28. Gao, W.; Gao, X.; Abtew, T. A.; Sun, Y.-Y.; Zhang, S.; Zhang, P. Quasiparticle Band Gap of Organic-Inorganic Hybrid Perovskites: Crystal Structure, Spin-Orbit Coupling, and SelfEnergy Effects. Phys. Rev. B 2016, 93, 085202.

29. Endres, J.; Egger, D. A.; Kulbak, M.; Kerner, R. A.; Zhao, L.; Silver, S. H.; Hodes, G.; Rand, B. P.; Cahen, D.; Kronik, L. et al. Valence and Conduction Band Densities of States of Metal Halide Perovskites: A Combined Experimental-Theoretical Study. J. Phys. Chem. Lett. 2016, 7, 2722-2729.

30. Smith, C. T.; Leontiadou, M. A.; Page, R.; O'Brien, P.; Binks, D. J. Ultrafast Charge Dynamics in Trap-Free and Surface-Trapping Colloidal Quantum Dots. Adv. Sci. (Weinheim, Ger.) 2015, 2, 1500088 .

31. Chen, K.; Barker, A. J.; Morgan, F. L. C.; Halpert, J. E.; Hodgkiss, J. M. Effect of Carrier Thermalization Dynamics on Light Emission and Amplification in Organometal Halide Perovskites. J. Phys. Chem. Lett. 2014, 6, 153-158.

32. Trinh, M. T.; Wu, X.; Niesner, D.; Zhu, X. Y. Many-Body Interactions in Photo-Excited Lead Iodide Perovskite. J. Mater. Chem. A 2015, 3, 9285-9290.

33. Wehrenfennig, C.; Liu, M.; Snaith, H. J.; Johnston, M. B.; Herz, L. M. Homogeneous Emission Line Broadening in the Organo Lead Halide Perovskite $\mathrm{CH}_{3} \mathrm{NH}_{3} \mathrm{PbI}_{3-\mathrm{x}} \mathrm{Cl}_{\mathrm{x}}$. J. Phys. Chem. Lett. 2014, 5, 1300-1306.

34. Chang, A. Y.; Cho, Y. J.; Chen, K. C.; Chen, C. W.; Kinaci, A.; Diroll, B. T.; Wagner, M. J.; Chan, M. K. Y.; Lin, H. W.; Schaller, R. D. Slow Organic-to-Inorganic Sub-Lattice Thermalization in Methylammonium Lead Halide Perovskites Observed by Ultrafast Photoluminescence. Adv. Energy Mater. 2016, 6, 1600422.

35. Zhu, X. Y.; Podzorov, V. Charge Carriers in Hybrid Organic-Inorganic Lead Halide Perovskites Might Be Protected as Large Polarons. J. Phys. Chem. Lett. 2015, 6, 4758-4761. 36. Frost, J. M.; Azarhoosh, P.; McKechnie, S.; van Schilfgaarde, M.; Walsh, A. A Photon Ratchet Route to High-Efficiency Hybrid Halide Perovskite Intermediate Band Solar Cells. ArXiv e-prints 2016, 1611.09786.

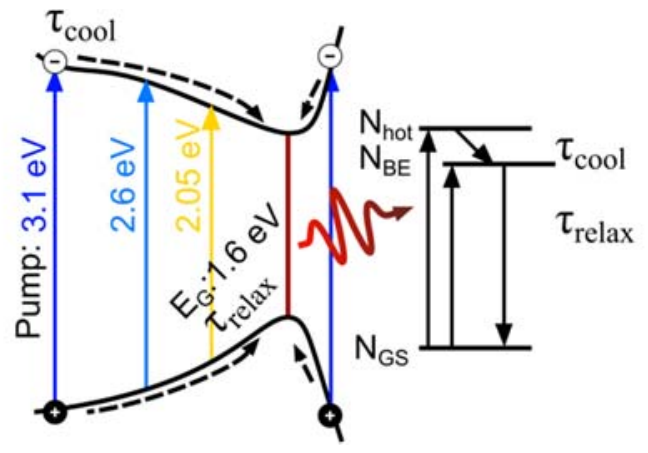

\section{TOC Graphic}

